\title{
Über einen Zusammenhang zwischen kritischer Temperatur und Ionisierungsspannung bei den Edelgasen
}

\author{
Von Erich Bagge und Paul Harteck

\begin{abstract}
Aus dem Kaiser-Wilhelm-Institut für Physik und dem Physikalisch-Chemischen Institut der Universität Hamburg
\end{abstract} \\ (Z. Naturforschg. 1, 481-482 [1946]; eingegangen am 1. Juni 1946)

\begin{abstract}
Zwischen der kritischen Temperatur und der Ionisierungsspannung besteht bei Edelgasen der durch Gl. (6) gegebene empirische Zusammenhang. Dies wird durch quantenmechanische Betrachtungen plausibel gemacht.
\end{abstract}

$\mathrm{D}$ as Auftreten der van der Waalsschen Anziehungskräfte zwischen Edelgasatomen, welche bei genügend tiefen Temperaturen zur Verflüssigung dieser Gase führen, ist heute durch die Quantenmechanik befriedigend geklärt1. Man kann die aus der Theorie abgeleiteten Ergebnisse etwa in folgender Weise zuisammenfassen: Zwischen zwei gleichartigen Edelgasatomen vom Abstand $R$ besteht in gewisser Näherung ein Wechselwirkungspotential der Form:

$$
V=A \exp (-R / a)-B / R^{6}-C / R^{8} .
$$

Die Konstanten, $A, B, C$ und $a$ sind für alle Edelgase bereits bekannt2. Das in Formel (1) auftretende Glied $A \exp (-R / a)$ bedeutet dabei ein Abstoßungspotential, dessen Wert in den äußersten Randbereichen der Atome durch die beiden letzten Glieder, welche ja einer Anziehung entsprechen. überkompensiert wird, so daß sich insgesamt eine schwache Anziehung dieser Teilchen ergibt.

Bei vorgegebenen Kräften zwischen den Atomen aber ist es - zumindest im Prinzip - möglich. die thermodynamischen Eigenschaften eines aus ihnen bestehenden Gases zu berechnen, wenngleich natürlich infolge der vor der Verflüssigung auftretenden Molekülbildung die Zusammenhänge be-

1 Wir verweisen auf die zusammenfassenden Darstellungen von H. M a r g e n a u, Rev. mod. Physics 11, 1 [1939] und F. L o n d o n, Trans. Faraday Soc. 33, 8 [1937], welche auch die Zitate der wichtigsten Originalarbeiten enthalten.

2 Siehe auch H. Jensen, Z. Physik 101, 164 [1936], and P. Go mb a s, ebenda 93, 378 [1935]. reits ziemlich verwickelt sind, und man in den meisten Fällen auf Näherungsrechnungen angewiesen ist.

Dieser Sachverhalt bringt es mit sich, daß trotz guter Kenntnis der Kräfte zwischen den Edelgasatomen nicht ohne weiteres alle Gesetzmäßigkeiten zwischen einfachen atomaren Eigenschaften und bestimmten thermodynamischen Zustandsgrößen zu erkennen sind.

Wir möchten nun im folgenden auf einen in der Natur vorhandenen sehr engen Zusammenhang zwischen einer solchen atomaren Eigenschaft, nämlich der Ionisierungsspannung $E_{J}$, und einer thermodynamischen Größe, der kritischen Temperatur $T_{k}$, hinweisen, der durch theoretische Betrachtungen zwar nahegelegt werden kann, der aber den bisherigen Theorien nicht unmittelbar zu entnehmen ist.

Wenn man nämlich versucht, die Anziehungskräfte zwischen den Atomen zu berechnen, so wird man daraufgeführt, den folgenden Ausdruck für die Störungsenergie 2. Ordnung zu bestimmen:

$$
E=\sum_{i=1}^{z} \sum_{n=1}^{z} \sum_{m=1}^{\infty} \sum_{n=1}^{\infty} \frac{\left|V_{00}^{m n}\right|^{2}}{2 E_{0}-E_{m}-E_{n}} .
$$

$\mathrm{V}_{00}^{m n}$ ist dabei das Matrixelement für die Coulo mbsche Wechselwirkung der Elektronen und des Kerns vom Atom 1 mit allen Ladungsträgern des Atoms 2, bei welchem im Atom 1 ein Elektron sich aus einem Zustande 0 in den angeregten mit dem Index $m$ begibt, während beim 2. Atom ein 
anderes Elektron gleichzeitig einen Übergang nach dem Zustand $n$ ausführt. In diesem Ausdruck tritt weiter auf die Energiedifferenz $2 E_{o}-E_{m}-E_{n}$, aus welcher die Energieänderung des Gesamtsystems bei diesen Übergängen zu entnehmen ist. Wie schon in vielen Arbeiten ${ }^{1}$ ausgeführt ist, kann man sie im Mittel für alle Übergänge, über welche die Summation bei den Edelgasen zu erstrecken ist, in guter Näherung einfach durch die doppelte Ionisierungsspannung des einzelnen Atoms ersetzen:

$$
2 E_{0}-E_{m}-E_{n} \approx-2 E_{J} .
$$

Die Wechselwirkungsenergie wird damit in dieser Näherung einfach umgekehrt proportional zur Ionisierungsspannung $E_{J}$ :

$$
E=-\frac{1}{2 E_{J}} \sum_{i=1}^{z} \sum_{h=1}^{z} \sum_{m=1}^{\infty} \sum_{n=1}^{\infty}\left|V_{00}^{m n}\right|^{2}
$$

oder nach einem bekannten Satze der Matrixmultiplikation:

$$
E=-\frac{1}{2 E_{J}^{\prime}} \sum_{i=1}^{z} \sum_{h=1}^{z}\left(V^{2}\right)_{00}^{0} 0
$$

wobei jetzt die Abkürzung ( $\left.V^{2}\right)_{0}^{00}$ den Erwartungswert für das Quadrat der Coulombschen Wechselwirkungsenergie beim Grundzustand der Atome darstellen soll, über dessen Größe ohne weitere Rechnungen keine Aussagen möglich sind. Immerhin aber wird man annehmen dürfen, daß die van d e r Wa a ls schen Anziehungskräfte in irgendeiner Weise noch einen Zusammenhang mit der Ionisierungsspannung der Atome erkennen lassen.

Ein sehr gutes Kriterium für die Stärke der van der Waals-Kräfte stellt nun der Wert der kritischen Temperatur dar. Es ist unmittelbar plausibel, daß die kritische Temperatur um so höher liegen wird, je fester die Atome sich gegenseitig binden können; dies wiederum sollte nach den obigen Darlegungen um so ausgeprägter zu bemerken sein, je geringer die Ionisierungsspannung ist.

Wir haben nun in Abb. 1 für die Edelgase die kritischen Temperaturen als Funktion der entsprechenden reziproken Ionisierungsenergien aufge-

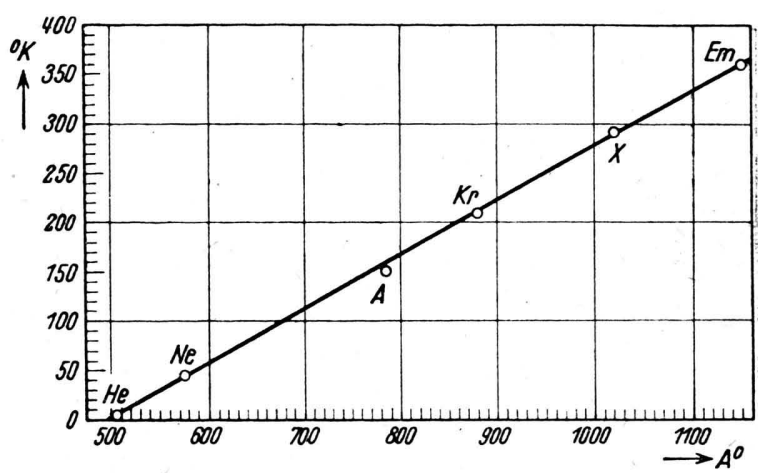

Kritische Temperatur der Edelgase als Funktion der reziproken Ionisierungsenergie $\left(A^{0}\right.$ bedeutet die der Ionisierungsspannung entsprechende Lichtwellenlänge in Angström-Einheiten).

tragen und stellen $\mathrm{zu}$ unserer eigenen Überraschung fest, daß alle Punkte sich auf einer Geraden anordnen. Man kann also den Satz aussprechen, daß bei den Edelgasen die kritischen Temperaturen sehr genau umgekehrt proportional $\mathrm{zu}$ den Ionisierungsspannungen sind. Formelmäßig läßt sich dieser Sachverhalt auch folgendermaßen ausdrücken:

$$
T_{k}\left({ }^{0} K\right)=-274,5+\frac{6860}{E_{J}(e V)} .
$$

Es ist nun Aufgabe der Theorie, die empirisch gut belegte lineare Beziehung (6) quantitativ zu. begründen. 